\title{
DESENCUENTROS ENTRE PRÁCTICAS ESCOLARES Y VIVENCIAS FAMILIARES
}

\author{
M.Sc. Teresita Cordero Cordero ${ }^{1}$
}

\begin{abstract}
Resumen: El interés de este ensayo es sustentar críticas que se hacen al sistema escolar formalizado, $y$ evidenciar prácticas de aprendizaje que la niñez adquiere en sus hogares. Las investigaciones referidas trabajaron con grupos culturalmente diferenciados, los cuales, tienen formas de enseñanza-aprendizaje particulares que no necesariamente se integran en la vida escolar; así se presenta el tema dentro de un proceso de continuidad-discontinuidad entre la vida familiar y la socialización escolar. De igual manera, se argumenta la dificultad que tiene el sistema educativo formal para integrar actividades culturales distintas, y se señalan formas de aprendizaje institucionalizadas de la escuela centrada en la figura del maestro. También se indican puntos de discusión que requieren atención de todos los participantes del proceso educativo, y se menciona la necesidad de que la escuela cambie e integre a las familias como actores; al tiempo que se crítica la rigidez de nuestras escuelas y el desconocimiento y desvalorización que se hace de los recursos sociales del mundo cultural, social y personal de los involucrados.
\end{abstract}

Abstract: The purpose of this essay is to issue the arguments for a list of critical commentaries about schools, and to demonstrate learning activities experienced by children at home. The aforementioned research dealt with groups of different cultural backgrounds, whose teaching and learning activities are not necessarily related to school life. This topic is presented within a process of continuity-discontinuity between family life and the schools' social environment. The formal education system's difficulty to introduce different cultural activities is discussed later. The school's institutionalized learning methods, centered on the teacher's role, are also pointed in the essay. The discussion topics that require attention by every participant of the learning process, as well as the need for the school to fit the family as performing parts in the learning process are both mentioned too. The lack of flexibility in our schools and of acknowledgement of social resources in the cultural, social and personal worlds of the people involved in this work is also criticized.

Palabras clave: ESTUDIOS SOCIO-CULTURALES / CONTINUIDAD-DISCONTINUIDAD ENTRE CASAESCUELA / INTEGRACIÓN CULTURAL / FAMILIA/ ESCUELA.

\section{Introducción}

La inadecuada integración de los grupos étnicamente diferenciados e inmigrantes al sistema educativo formal, es un buen ejemplo de una problemática educativa a la que no se ha podido dar una respuesta certera. De igual manera, estudios socio-culturales demuestran la necesidad de realizar una serie de cambios en las escuelas, señalando la pérdida de recursos culturales de amplios sectores de la población en la vida cotidiana de la escuela. Lamentablemente, son muy pocas las experiencias que han roto con la rigidez del sistema educativo.

\footnotetext{
${ }^{1}$ Master en Psicología y candidata a doctora por el Programa Latinoamericano de Educación en la Universidad de Costa Rica. Desde 1991 es profesora en la Escuela de Psicología de la UCR, ocupa el puesto de subdirectora en la misma Escuela y es investigadora en el Instituto de Investigación de Mejoramiento de la Educación Costarricense (IIMEC) de la UCR. Ha ocupado los puestos de coordinadora de Docencia de la Escuela de Psicología, profesora investigadora en el Instituto de Investigaciones Psicológicas (IIP). Se ha desarrollado en el campo de la Psicología Social Comunitaria y actualmente esta estudiando los temas de desarrollo infantil. estudios culturales y educación.

e-mail: tcordero@cariari.ucr.ac.cl
} 
En la actualidad, es posible encontrar estudios que plantean diversas posiciones, debates y experiencias prácticas que dan cuenta de la discriminación, rechazo, asimilación o integración de grupos diferenciados en contextos escolares (Merino \& Muñoz, 1998).

El Consejo Europeo, en el año 1986 (Muñoz 1998), definió una serie de líneas de atención y respuesta a esta situación. Se estableció que los grupos culturales son una riqueza social justamente por su diversidad; asimismo, confirmaron la necesidad de respetar la especificidad y destacaron la importancia de promover la igualdad de oportunidades, ampliando el margen de acción no solo a los grupos étnicos diferenciados, sino a toda la población.

Esta posición reta al sistema educativo para que se abra y se sensibilice a la diversidad cultural que usualmente se hace invisible en la dinámica cotidiana de las aulas escolares (Hernández, 2000). Un conocimiento comprensivo del bagaje socio-cultural de los participantes que forman parte de la vida escolar, apelaría a ejecutar cambios importantes a la modalidad de enseñanza-aprendizaje (que por lo general se centra en la figura del docente) rompiendo con prácticas excluyentes. La escuela como institución, tiene que renovar sus prácticas, hábitos y costumbres, para construir experiencias equitativas y solidarias.

El presente trabajo tiene como objetivo rescatar algunos estudios culturales que señalan y muestran la problemática que existe entre las prácticas culturales que los niños y las niñas han aprendido en sus hogares, pero que no son tomados en cuenta en la escuela. La presente revisión retoma de manera especial estudios etnográficos que dan cuenta de dichas prácticas sociales en grupos diferenciados, de aquellos que han asumido las actividades dominantes]

La familia y la escueld son dos de las instituciones sociales que estructuran la vida humana. Sus prácticas cotidianas obedecen a patrones o actividades que trasmiten estilos de relación interpersonal y formas de organización social. Desde una perspectiva sociohistórica, la vida mental se sustenta en las experiencias que se han desarrollado en el

\footnotetext{
${ }^{2}$ Rogoff (1993) ubica en los grupos dominantes norteamericanos a las personas blancas de clase media y de origen europeo.

${ }^{3}$ Ambas instituciones tienen múltiples maneras de conformarse; no obstante, en este ensayo se habla de en términos generales, sin entrar en una discusión pormenorizada de cada una.
} 
mundo social. Por tal razón, el aprendizaje es una actividad social y no una acción individual. Así, el acto social se convierte en una precondición de la conciencia individual y toda actividad humana es un reflejo de la cultura y de la historia del grupo(s) al cual se pertenece (Wertsch, 1995; Rueda, Genzuk, Baca y Hentschke, (s.f.); Chavés, 2001).

Los estudios revisados de Health (1982), Philips (1982), Delgado-Gaitán (1987 y 1994), Matusov y Rogoff (en prensa), Matusov, Bell \& Rogoff (2002), Rogoff, Turknis \& Bartlett (2001) y Hernández (1998, 1999, 2000), son trabajos que cuentan con años de contacto directo con las poblaciones estudiadas y todos utilizan técnicas diversas en ambientes naturales. Por ejemplo, los trabajos de Health (1982), Philips (1982), Delgado-Gaitán (1987 y 1994) y Hernández (1998, 1999, 2000), son de corte etnográfico.

La escogencia de dichas investigaciones no ha sido casual, por el contrario se consideró que la ventaja está en la comprensión y el análisis de los procesos de socialización de distintos grupos sociales en el contexto familiar y escolar.

De manera adicional, se refieren otras investigaciones dentro del campo de los estudios socio-culturales que complementan algunos argumentos, tales como los de Matusov y Rogoff (en prensa) y Matusov, Bell \& Rogoff (2002), cuyas investigaciones forman parte de un amplio programa en el campo del desarrollo infantil y la educación. Estos estudios registran en video-tape el momento y la forma en que se realizan ciertas tareas específicas. Posteriormente, los registros son sometidos a un proceso de análisis de casos, y por medio de "jueces" externos, se codifica la información. Los casos son comparados o agrupados según alguna característica en particular que distingue a los actores. Se agrupan similitudes y diferencias llegando a conclusiones que dan pie a nuevas investigaciones.

En el caso de Hernández $(1998,1999,2000)$ los trabajos ya publicados se enriquecerían aún más con la información que el autor tiene sin publicalten cuanto a las prácticas familiares.

\footnotetext{
${ }^{4}$ De acuerdo con la conversación informal con el autor (marzo, 2003).
} 


\section{Papel de las instituciones}

El papel de la familia sigue siendo importante, sobretodo porque mantiene la especie humana e incorpora a los recién nacidos a la vida social. Como grupo social primario, la familia se convierte en el espacio más cercano e íntimo, que de manera ideal, apoya y promueve el desarrollo infantil. Los infantes, al participar en la vida familiar, incorporan y aprenden las prácticas culturales del grupo al cual pertenecen y por medio de las que aprenden cómo ser en el mundo.

No obstante la importancia que tiene la familia en la socialización humana, hay condiciones políticas y sociales que están tratando de incorporar a la niñez a la institucionalización escolar tempranamente; objetivo que busca instrumentalizar y regular la vida en sociedad acorde con el sistema económico que impera, cuyo criterio principal es el ajuste de los niños y de las niñas, de manera temprana, a los requerimientos que la escuela y el sistema social demandan.

Es interesante mencionar cómo los gobiernos latinoamericanos, apoyados por los organismos internacionales, han oficializado la educación preescolar, argumentando una reducción de los índices de deserción y repitencia escolał Quizá, uno de los problemas más serios de este proceso, es el que plantea Myers (1995), cuando alerta sobre el peligro de perder la riqueza y la flexibilidad de las experiencias de la educación temprana, escolarizando al preescolar.

La institución escolar occidental se consolida, durante el siglo XX, como una de las instituciones más importantes de socialización humana (Rogoff, 2002). El modelo económico de producción y de acumulación de capital contribuye plenamente a fortalecer dicha institución. Las empresas necesitan trabajadores productivos y capaces de manejar los requisitos que le imponen los procesos de globalización e industrialización (Gutiérrez, 1997; Vargas, 1996; Wing-Ching, 1998).

Por tal razón, la escuela tiene la tarea explícita de instruir a los seres humanos en aquellas herramientas que la cultura occidental dominante considera fundamentales tales como la lectura, la escritura, la matemática y algunos conocimientos generales de la ciencia

\footnotetext{
${ }^{5}$ Visión simple de una problemática mucho más compleja y que de ninguna forma se resuelve por el aumento de años escolares, lo que requiere de una discusión que no se aborda en este trabajo.
} 
y la tecnología, básicos para responder al modelo dominante de desarrollo económico-social. De manera adicional, la escuela se encarga de transmitir y enseñar un modelo de relación humana basado en la jerarquía y el poder centralizado en una jefatura que tiene la autoridad para definir y organizar las relaciones sociales, acorde con los requerimientos sociales (Philips, 1983; Delgado-Gaitán, 1987; Health, 1982; Mutosov, Bell \& Rogoff, 2002 y Hernández, 2000).

El modelo que prevalece en la escuela occidental está centrado en el adulto, pues la autoridad del maestro es prácticamente absoluta. El docente es quien organiza todas las actividades y define las formas de comunicación permitidas en el aula (Philips, 1982; Health, 1982 \& Matusov, Bell \& Rogoff, 2002), dejando de lado la posibilidad de la cooperación y colaboración entre los demás niños y niñas o familiares, así como los diversos estilos de aprendizaje.

\subsection{Continuidad-discontinuidad}

A partir de un amplio espectro de acción aprendido en la vida familiar, la niñez acumula un bagaje de prácticas que su cultura considera valiosas. Sin embargo, cuando el niño o la niña pasa o transita de la familia a la escuela, surgen diferencias que encaran en una problemática. Esta situación, para algunos autores norteamericanos, se ha nombrado como los procesos de continuidad-discontinuidad[ concepto que requiere un análisis cuidadoso para puntualizar mejor una posición.

Es interesante que, si se ubica el énfasis en el contexto escolar tradicional, se pondría también el acento en la discontinuidad. Argumentos varios se esgrimen a favor de esta posición; por un lado, porque si el infante no se adapta a las demandas de la escuela, se presentan son deficiencias en las habilidades y destrezas, cuya connotación es la existencia de una privación cultura $\mathbf{\square}$ Visto de esta manera, la posición es etnocéntrica, pues supone que hay una sola manera de aprender y que toda actividad diferente, que no calza con lo dominante o establecido se considera inválido, erróneo o inadecuado.

\footnotetext{
${ }^{6}$ Idea que surge originalmente de los estudios antropológicos de los años sesenta, que mantenía la concepción de que existen culturas deficitarias que no ofrecen los aprendizajes adecuados para que los individuos logren el éxito (Ogbu, 1982), o como se le conoció en América Latina, la cultura de la pobreza, que trae una carga ideológica determinista y excluyente.

7 Término bastante discutido y cuestionado por el determinismo, y que involucra desvalorización de formas distintas de hacer las actividades sociales.
} 
Esta descalificación hacia prácticas culturales diferentes, trae consecuencias directas en la población infantil; y quizá una de ellas es el fracaso escolar. La solución que el sistema educativo plantea es el ajuste de la niñez a la escuela, sin reflexionar más allá de las rutinas ya institucionalizadas. Esta posición parece estar mayoritariamente difundida, y en algunos casos reforzada por los propios formadores universitarios en ámbitos como la Educación y la Psicología, entre otros.

Por otro lado, se puede hacer una lectura opuesta a la anterior. La discontinuidad que el niño o la niña vive en la escuela no es producto de su incapacidad para aprender, sino que es un problema del sistema educativo formal, el cual, no logra integrar la riqueza cultural que se trae. Por lo tanto, la institución escolar tendría que incorporar nuevas modalidades de enseñanza-aprendizaje que tomen en cuenta el potencial socio-cultural que los estudiantes ya tienen. Tal cual lo exponía Bottani en 1983, cuando discutía desde el campo de la educación preescolar, " . . . No se llegará a ningún cambio sustancial en el campo de la educación infantil temprana si insistimos en cambiar a los niños en vez de comenzar a cambiar la escuela" ( p. 156)

En este mismo sentido, en Costa Rica, Hernández (2000) ha señalado esta misma dificultad en las escuelas costarricenses; las cuales, desaprovechan los recursos culturales de sus alumnos. Lamentablemente, la tendencia general es sustituir los recursos de los lugareños por métodos y materiales descontextualizados, distantes y sin sentido para el referente cultural identitario de los estudiantes. De igual forma el autor demuestra que existe un rito de iniciación que impone una barrera simbólica que excluye la participación de las familias en el aula escolar.

Desde esta posición, se plantea la necesidad de impulsar nuevas y alternativas formas de educación que incorporen prácticas diferentes, y que fomenten la creación de comunidades de aprendices $\mathbf{E} q u e$ apoyen al desarrollo humano. Una experiencia educativa innovadora que ya da cuenta de esta propuesta es la que han desarrollado Rogoff, Turkanis

\footnotetext{
${ }^{8}$ La comunidad de aprendices hace referencia a un ambiente donde se reconoce que el aprendizaje es producto de la convivencia social. Así, todos los participantes de diferentes edades y condiciones aprenden y enseñan unos de los otros, al poner en común la experiencia y las prácticas culturales cotidianas. La comunidad de aprendices tiene la característica de que transforma a quienes participan. La forma de trabajo está basada en la cooperación y la participación mutua. Hemos de recordar la propuesta aún vigente de Paulo Freire (1969) “. . . que nadie educa a nadie; que tampoco nadie se educa sólo: que los hombres se educan entre sí, mediatizados por el mundo ... ." (Freire, 1969 pp. 18).
} 
\& Bartlett (2001), quienes integran a las madres y padres de los niños y de las niñas en la práctica de trabajo cotidiano en una escuela primaria pública en Utha, Estados Unidos.

\subsection{Ejes de análisis en la familia}

Los estudios revisados sobre la vida familiar proviene de las siguientes poblaciones: indígenas norteamericanas, afro-americanas, inmigrantes mexicanos; blancos de la clase media, así como negros, mestizos e indígenas de la zona atlántica costarricense.

Quizá, uno de los ejes coincidentes en los estudios, es lo que Rogoff (1993) sintetiza como el aprendizaje de relaciones de interdependencia y cooperación que se dan en los hogares, versus el aprendizaje de la independencia que se fomenta en las clases medias de origen europeo en Los Estados Unidos de América. Las relaciones entre hermanos y familiares se convierten en el eje de las relaciones al interior de las familias. La negociación y el contacto interpersonal son básicos en las prácticas cotidianas (Rogoff, 1993, en prensa; Delgado-Gaitán, 1987 y 1994, Matusov, Rogoff, en prensa; Matusov, Bell \& Rogoff, 2002).

La cooperación-competencia es, por lo tanto, un eje de análisis utilizado en las investigaciones, y da cuenta no sólo, de una conducta en particular, sino que de un conjunto de habilidades y destrezas que cobran sentido al interior de la vida del grupo y del contexto social vivido. Los estudios referidos en este artículo, presentan una visión distinta de las investigaciones realizadas en los años setenta, las cuales estuvieron interesadas en demostrar que tanta cooperación o que tanta competencia se producía en ambientes experimentales Por el contrario, los estudios presentan y amplían la idea de la existencia de una red de sentido que transforma la vida en sociedad y que apoya la vida cotidiana, estructurando redes de apoyo para la supervivencia y convivencia social.

Así, las formas de comunicación que se establecen corresponden más a intercambios cercanos donde se enfatiza la observación y el contacto físico, Philips (1982) describe que la mirada es intensa en la comunicación no verbal de los indígenas norteamericanos, y Health (1982), por su parte, explica que la expresión oral de los grupos afro-americanos, especialmente cuando cuentan, comparten historias y participan de las conversaciones colectivas en la cotidianidad familiar, son fundamentales en el aprendizaje. Hernández

${ }^{9}$ Shapira; Madsen (1969); Madsen \& Shapira (1970); Thomas, (1975); Hollos, (1980) 
(2000) insiste en la importancia de incorporar la tradición oral a la práctica escolar, actividad que no se desarrolla ni fomenta en las aulas de la zona atlántica costarricense.

Si se hace un contraste entre las formas de comunicación establecidas en el hogar versus la escuela, en la familia se registran menos preguntas directas y más afirmaciones en contexto (Health, 1982; Philips, 1982). Las preguntas directas, para Herts-Lazarowitz \& Shachar (1990), expresan distancia en las relaciones y reducen la espontaneidad, la intimidad y la creatividad.

Las relaciones de autoridad que se reportan en las familias de origen mexicano en el estudio de Delgado-Gaitán (1987) y en el pueblo indígena norteamericano (Philips, 1982) así como en los grupos afro-americanos (Health, 1982), se constituyen a partir de una red de relaciones sociales que parten de los vínculos entre hermanos y familiares cercanos. El control de las conductas infantiles se da en un contexto colectivo, y los niños y las niñas aprenden a ser autosuficientes y a asumir responsabilidades que se consideran aptas para su edad.

Muchos de estos aspectos difieren de los estudios sobre las familias blancas de clase media norteamericana, quienes más bien tienden a reproducir un estilo de relación más cercano al sistema escolar (Rogoff 1993, 2002), y mantienen el control y el poder en los adultos, quienes a su vez estructuran y definen los ámbitos de acción de la niñez.

Dichas prácticas sociales de contactos interpersonales diversos, se convierten en un ambiente enriquecido para el desarrollo infantil. Lastimosamente, como concluye DelgadoGaitán (1987), muchas de las habilidades y destrezas que los niños y las niñas ya poseen no son reconocidos como valiosos, y suelen chocar en el marco de la enseñanza formal. Esta discontinuidad crea la sensación de incapacidad infantil y sin temor a equivocarse, se encasillan en diagnósticos tales como "problemas de aprendizaje", "atrasos en el desarrollo" o "deficiencias personales" para el aprendizaje. La exclusión que pueden vivir la niñez en la escuela se interpreta como fracaso escolar, cuya connotación social es negativa. Surge con fuerza la idea de la privación cultural argumento que discrimina y frustra intentos legítimos de grupos sociales diferentes. 
Las relaciones familiares diversas en estos grupos, tienen la característica de que se les exige a la niñez desarrollar la capacidad de observar, escuchar y poner atención de manera simultánea o tomando en cuenta múltiples actividades a la vez (Delgado-Gaitán, 1987, Chavajay y Rogoff, 1999), aspecto interesante que suele estar opuesto al ambiente escolar, donde se exige la atención centralizada en un solo evento. Desde esta información, habría que cuestionar muchos de los diagnósticos de "hiperactividad" o de "personas con déficit de atención", que suelen hacerse en el contexto escolar ¿No será más bien que la escuela no ha desarrollado otras formas para permitir desarrollar la atención múltiple en vez de censurarla y castigarla?

\subsection{Ejes de análisis en el ambiente escolar}

En la escuela la diferencia de roles está claramente establecida. El adulto especialista, el docente, funciona desde la jerarquía y tiene el poder de decidir sobre todo lo que se realiza en el contexto institucional. De igual manera, el educador tiene el poder de la palabra y de la comunicación. Por ejemplo, Philips (1982) comenta que la maestra, cuando se dirige a todo el grupo, espera que haya una respuesta a coro. El docente puede cambiar la estrategia y llamar a cada estudiante uno por uno, turnando el uso de la palabra, pero siempre la comunicación se caracteriza en función del adulto autorizado. Concluye Philips (1982) que en el aula escolar, la comunicación permitida y fomentada es entre el alumno y la maestra. En este ambiente queda excluida la relación entre compañeros, por tal razón, la conversación y la ayuda de parte de otros niños y niñas suele ser castigada en el contexto de "hacer trampa", y es vista como indisciplina.

Desde una perspectiva socio-cultural, se plantearía todo lo contrario, la participación, el compromiso y la ayuda que se dan los niños y las niñas en el aprendizaje, es fundamental. En este sentido, habría que preguntarse si los espacios informales de la escuela, como los recreos, son los más apreciados por los alumnos que el tiempo destinado en el aula, el cual puede ser vivido como un ambiente empobrecido.

A pesar de que en la escuela hay trabajo en pequeños grupos, éstos tienden a repetir el modelo escolar. La autoridad de la maestra estimula la presencia de un "líder" que tiene la obligación de controlar y organizar la acción del resto del grupo. Delgado-Gaitán (1987) agrega que en el aula escolar los niños y las niñas son agrupados de acuerdo con el 
interés que la docente define, produciendo mayor competencia entre los pares y poca colaboración y apoyo solidario entre los alumnos.

El estudio de Matusov, Bell \& Rogoff (2001) demuestra cómo el modelo que se práctica tiende a repetirse. En el estudio que realizaron, los niños y las niñas que provenían de una enseñanza cooperativa buscaban compartir sus ideas con el compañero de trabajo, pero los infantes procedentes de una escuela tradicional repiten las prácticas culturales centradas en el adulto, hacen pequeñas pruebas y orientan la acción por medio de preguntas, sin considerar ni involucrar a su compañero en la solución de las tareas encomendadas.

Asimismo, en una gran parte del tiempo en el aula se fomenta el trabajo independiente凹 para que el alumno realice por sí solo la tarea. Esta modalidad de trabajo excluye cualquier tipo de ayuda y cooperación entre compañeros y crea la imagen que ayudarse entre sí, significa hacer "trampa", por lo cual es una conducta negativa (DelgadoGaitán, 1987; Matusov, Bell \& Rogoff, 2002).

Otro ejemplo de cómo la modalidad centrada en la autoridad del adulto se reproduce, son las reuniones con madres y padres de familia que reporta Hernández (1999). Este autor detalla cómo el docente asume el protagonismo. La autoridad está en el especialista, quien a su vez, juzga y sentencia el actuar de las madres y padres o encargados. La idea es que los familiares son los principales responsables de la educación de la niñez, con la paradoja y contradicción que ellos como grupo no pueden intervenir en los procesos de enseñanzaaprendizaje de sus hijos e hijas dentro del aula. Así, prevalece la interpretación del maestro sobre la interpretación de las familias.

Cuando los niños y las niñas ingresan a la escuela se marca y establece una barrera simbólica entre ambas instituciones sociales. Otros adultos "especialistas" tienen la tarea de enseñar, envestidos en la figura de la maestra y las relaciones se fijan no por el afecto, sino por el conocimiento y el control (Hernández, 2000).

\footnotetext{
10 En Costa Rica se encuentran textos escolares, llamados de "trabajo independiente", y que son utilizados frecuentemente en la escuela.
} 
A su vez, Hernández (2000) señala de manera controversial, que los métodos de enseñanza no son razonablemente comprendidos por los propios educadores sigue siempre una sola vía para su instrucción. La diferencia la marcan los docentes más creativos, los cuales, utilizan una posición ecléctica de la enseñanza de la lecto-escritura, por ejemplo. Hernández (2000) interpreta este déficit como una consecuencia del escaso ejercicio crítico que hacen los maestros de los materiales didácticos, la poca investigación que realizan y las medidas restrictivas que impone el Ministerio de Educación Pública.

El ejercicio docente, por su parte, no promueve en los estudiantes la investigación ni la comunicación de su propia cultura. Hernández (2000) puntualiza cómo el material cultural y simbólico de la comunidad esta ausente. Señala que el aprestamiento es un ejemplo de la ruptura y distanciamiento que propicia la escuela con respecto a la historia de origen de sus alumnos. El modelo educativo considera que los niños y las niñas están vacíos, por tal razón, la tarea escolar es llenarlos. En casi ninguna ocasión la escuela se adecua a la identidad de sus estudiantes y docentes, violentando procesos sensibles para todos los participantes.

En un intento de homogenizar a los participantes, en Costa Rica, explica Hernández (2000), la escuela utiliza el uniforme para ocultar las diferencias que estructuran la vida comunal. La escuela trata de homologar al estudiante y al personal, aunque en la dinámica de relaciones escolares, se muestran las diferencias étnicas y socioeconómicas a través del discurso, el uso de la tecnología, la expresión corporal y el acceso a recursos. Los infantes tienen que aprender otras formas de relación y actuación que no son propias de la vida familiar, con la consecuente dificultad para mantenerse como educando. ¿Será está una de las razones de la deserción escolar en nuestro país?

\subsection{Resultados de los trabajos y algunas comparaciones}

En el aula, los maestros tienen la tarea de instaurar un estilo de relación donde el control está bajo la responsabilidad del adulto. Por lo general, se valora una escuela positivamente cuando todos los alumnos están en silencio realizando su trabajo independiente sin más posibilidad de conversar y responder a las preguntas que hace la maestra. En este sentido cuando se piensa en la probabilidad de encontrar una escuela donde la niñez escoja qué hacer en grupos pequeños de colaboración mutua, y con un currículo flexible y abierto al diálogo y la cooperación, se denota el lugar como "anárquico", lo 
que conlleva el peligro de no enseñar lo que se supone el currículo Rogoff, en prensa; Rogoff, Turkanis, Bartlett, 2001). La opción más tentadora, y la que prevalece, es mantener el orden y el control por sobre las necesidades de los estudiantes, creando las condiciones óptimas para la exclusión. Por tal razón, se afirma que la escuela mantiene una estructura de relación rígida y autoritaria que atrapa tanto a estudiantes como a docentes.

La escolarización en occidente incentiva la competencia y el aislamiento más que la cooperación (Rogoff, 2002) en contraste con la vida de las familias estudiadas, provenientes de grupos sociales minoritarios y pobres que fomentan la cooperación y la ayuda mutua. Como se puede constatar en los estudios, los niños y las niñas de estas familias suelen colaborar, negociar y participar en conjunto con sus parientes en un marco de acción colectiva.

Cuando se ingresa a la escuela y se plantea que es necesario que los niños y las niñas conozcan y aprendan los contenidos básicos de ciertas materias (lectura y escritura, matemática y ciencias), no existe duda de que esto les ofrece mayores competencias para enfrentar al sistema económico actual; el problema está en cómo se lleva a cabo dicha tarea y la forma en que se instaura. Lamentablemente, la escuela aún no logra integrar prácticas creativas y oportunas que sean significativas para los alumnos y por ello, las estadísticas siguen demostrando que el fracaso de los escolares es una crisis del sistema educativo formal.

Conocer más sobre la forma en que la diversidad juega en los grupos sociales y reflexionar sobre ello, es una necesidad y una responsabilidad que cada día es más evidente en la práctica profesional de los docentes. Este trabajo es sencillamente un llamado de atención a continuar con esta discusión, y a profundizar sobre el tema con el interés de crear formas alternativas, que puedan superar los problemas mencionados.

Lógicamente, solucionar esta problemática implicaría cambiar años de socialización que se han internalizado en modelos de relación y organización social que están como lo dice Philips (1982) ". . .en medio de una cultura invisible". En este sentido, Matusov \& Rogoff (2002) y Rogoff, Turknis \& Bartlett (2001), demuestran que el cambio de un modelo

\footnotetext{
${ }^{11}$ El tema del currículo merece, en otra oportunidad, un estudio más crítico y reflexivo.
} 
de enseñanza centrado en el adulto, a uno de participación mutua, requiere de años, pues se necesita romper con una serie de esquemas que están plasmados en las actuaciones más que en los discursos.

\section{Algunas reflexiones}

Nuestros sistemas educativos han desarrollado un estilo de enseñanza que separa a las familias de la institución escolar y que jerarquiza las relaciones sociales (Matusov, et.al 2002, Rogoff, en prensa, Rogoff, Goodman Turkanis, \& Bartlett 2001). La incorporación y la participación de los padres en las escuelas como agentes educativos activos, implica un cambio en las prácticas culturales en que la escuela parece no estar interesada. Se constituye en un reto para la sociedad ofrecer respuestas significativas a las necesidades que no son solo demandas de la población escolar sino de los propios maestros.

Los ejes de cooperación y ayuda mutua se han convertido en una estrategia de supervivencia humana, que a su vez son un valor social para muchos grupos humanos. Los procesos de industrialización y la tecnificación de la vida humana generan formas de relación distantes que responden a las necesidades de un sistema económico que deshumaniza la vida en sociedad. Este es un grave peligro que atenta contra el derecho a la identidad, así como contra el respeto de prácticas culturales propias y diferentes.

América Latina necesita conocer mejor sus experiencias culturales autóctonas y urgentemente, se deben articular maneras creativas de enseñanza-aprendizaje flexibles y oportunas. En este sentido, conocer los estudios empíricos ofrece la oportunidad de encontrar formas de acercamiento que podrían servir para realizar una enseñanzaaprendizaje pertinente.

Quizá, una de las vías para lograr ese objetivo, sea un cambio en el sistema educativo formal que impulse otros modelos de enseñanza donde la participación y la cooperación sean los medios y no la excepción. La idea de crear comunidades de aprendices, en las que participen activamente las familias y los niños y las niñas, y en que la escuela propicie un ambiente que facilite el encuentro entre los participantes. Esto no necesariamente es una utopía ya se viene demostrando que es posible hacerlo (Rogoff, Matusov \& White, 1996; Rogoff \& Chikako, 1997 ; Rogoff, 1994; 2002; Rogoff, Turknis \& Bartlett, 2001). 
Los maestras y las familias tienen la tarea de estudiar y proponer una estructura escolar más humana que se resista a los intereses de orden económico y político que prevalecen en la actualidad. Desde esta posición y en el marco de finalizar con una "lluvia de ideas", se puntualiza con las siguientes recomendaciones:

1. Iniciar un proceso de discusión y análisis, que de manera participativa involucre a maestros(as), niños y niñas, madres y padres en el tema del desarrollo educativo.

2. Cuestionar el modelo educativo centrado en el adulto.

3. Sensibilizar a los docentes acerca de la importancia de conocer la vida de sus alumnos. Para ello, el conocimiento de las familias y la historia de los pueblos o comunidades es fundamental.

4. Integrar la vida personal, familiar y comunitaria como una fuente de aprendizaje dentro del mundo escolar, y no solo verla como un elemento folclórico.

5. Incorporar la escuela a las actividades de los lugareños, integrando acciones conjuntas que apoyen objetivos comunes.

6. Incluir la participación familiar en un marco de horizontalidad y respeto y no como fuente de mano de obra barata. Valorar el recurso humano aún a pesar de las diferencias económicas, éticas y sociales.

7. Fomentar las relaciones de cooperación entre los niños y las niñas de diferentes edades, para potenciar así el desarrollo humano.

Descubrir lo que la niñez ya tienen dentro de sí, y construir comunidades de aprendices para potenciar sus aprendizajes, incluyendo más que excluyendo, parece ser una utopía que no le interesa potenciar al sistema educativo formal, pues se pierde "el control" y el poder. Pero si no se hace, la escuela como institución tenderá a desaparecer. 


\section{Referencias}

Bottani, Norberto. (1983). De niño a alumno: ¿Ganando el Juego pero Perdiendo el Partido?. En Centro Internacional de Investigaciones para el Desarrollo, CIID; Evitando el fracaso escolar: relación entre la Educación Preescolar y la Primaria, informe del seminario sobre investigación preescolar celebrado en Bogotá, Colombia, mayo 26-29, 1981. Ottawa, Ontario, Canadá: CIID, pp. 146-157.

Chavajay, Pablo; Rogoff, Barbara. (2000). Schooling and Traditional Collaborative Social Organization of Problem Solving by Mayan Mothers and Children; Schooling and Social Organization.

Chavés, Salas, Ana Lupita. (2001). Implicaciones educativas de la teoría sociocultural de Vigostsky. Revista de Educación, 25, 2. pp.59-65.

Delgado-Gaitán, Concha. (1987). Traditions and transition the learning process of Mexican Children: an Ethnographic View. En: Spindler, George and Louise; Interpretive ethnography of education: at home an Abroad. New Jersey: Lawrence Erlbaum Associates, Publishers.

Delgado-Gaitán, Concha. (1994). Socializing Young Children in Mexican-American families an intergenerational perspective. In: Greenfiel, Patricia \& Cocking, Rodney (Eds); Cross-cultural roots of minority child development. USA: Lawrence Erlbaum Associates, Publishers.

Freire, Paulo. (1969). La educación como práctica de la libertad. Argentina: editorial Siglo $\mathrm{XXI}$.

Gutiérrez, Salomé. (1997). La nueva educación. Reflexiones, 59, junio.

Health, Shirley Brice. (1982). Questioning at home and at school: a comparative study; En: Spindler, George (edited by). Doing the ethnography of schooling, education anthropology in action. USA: CBS, College Publishing. (pp 102-131).

Hernández C., Omar. (1998). Micropolítica escolar hacia afuera: lecturas de una escuela Caribeña costarricense en la interacción con la comunidad y la familia. Revista de Educación, 22, 2. pp. 7-23.

Hernández C. Omar. (1999). Concepciones, disposiciones y prácticas hacia el capital escolar en un poblado liniero del Caribe costarricense. Revista de Educación, 23, 2. pp. 175-188.

Hernández C. Omar. (2000). Rituales de iniciación escolar. El caso de una escuela caribeña costarricense. Revista de Educación, 24, 1. pp. 61-76.

Hertz-Lazrowitz, Rachel. (1990). Teachers' verbal behavior in cooperative and whole class instruction. In Shara, Shlomo. Cooperative learning, theory and research. Greenwood Press, Inc.

Hollos, E. S. (1980). Collective education in Hungary: The development of competitive, cooperative and role-taking behaviors. Ethos, 8, 3-23. 
Madsen, M. C. \& Shapira, A. (1970). Cooperative and competitive behavior of urban AfroAmerican, Anglo-American, Mexican-American, and Mexican village children. Development Psychology, 3, 16-20.

Matusov, Eugene; Bell, Nancy \& Rogoff, Barbara. (2002). Schooling as cultural process: Working together and guidance by children from schools differing in collaborative practices. In R.V., Kail \& H. W. Reese (Eds). Advances in child development and behavior, 29, (pp. 129-160) San Diego, CA: Academic Press.

Matusov, Eugene; Rogoff, Barbara; (en prensa); Newcomers and Oldtimers: Educational Philosophies-in-Action of Parent Volunteers.

Merino, Fernández, J.; \& Muñoz, Sedano, Antonio. (1998). Ejes de debate y propuestas de acción para una pedagogía intercultural. Revista Iberoamericana de Educación, 17, pp. 207-247.

Muñoz, Sedano, Antonio. (1998). Hacia una educación multicultural: enfoques y modelos. España: Revista Complutense de Educación, 9, 2, 101-135.

Myers, Robert, G. (1995). La Educación Preescolar en América Latina, el estado de la práctica. Santiago, Chile: PREAL. Material retomado de los documentos en la red de internet, específicamente en la sección de documentos completos, de Red de Educación REDuc. Búsqueda realizada en noviembre 2000.

Oglu, John. (1982). Cultural discontinuities and schooling. In Anthropology \& Education Quarterly, 23, 4, 290-307.

Philips, Susan Urmston. (1983). The invisible culture, communication in classroom and community on the warm springs Indian reservation. USA: Longman Inc.

Rogoff, Barbara. (1994). Developing Understanding of the Idea of Communities of Learnes. Mind, Culture, and Activity, 1, 4 Fall.

Rogoff, Barbara; Matusov, Eugene; White, Cynthia. (1996). Models of Teaching and Learning: Participation in a Community of Learners. Handbook of education and human development. Oxford, UK: Blackwell.

Rogoff, Barbara y Toma, Chikako. (1997) Shared Thinking: Community and Institutional Variations. Ablex Publishing Corporation. Discourse Processes, 23, 471-497.

Rogoff, Barbara; Turknis, Goodman, Carolyn \& Bartlett, Leslee. (Eds) (2001). Learning Together, Children and Adults in School Community. USA: Oxford University press.

Rogoff, Barbara \& Angelillo, Cathy. (2002). Investigating the coordinated functioning of multifaceted cultural practices in human development. In: Human Development, 45, pp. 211-225.

Rogoff, Barbara. (2002). The cultural nature of human development. Oxford University Press, fothcoming. 
Rueda, R., Genzuk, M., Baca, R. \& Hentschke, G., (s.f). Latino paraeducators as teachers: Building on funds of knowledge to improve instruction. University of Southern California, Center for Research on Education Diversity and Excellence, (CREDE), final report, proyect 2.3

Shapira, A., \& Madsen, M. C. (1969). Cooperative and competitive behavior of Kibbutz an urban Children in Israel. Child Development, 40, 609-617.

Vargas, Alicia E. (1996). Globalización, capital humano y educación. Reflexiones, 52, noviembre.

Wertsch, James. (1995). Vygotsky y la formación social de la mente. España: Editorial Paidós.

Wing-Ching, Isabel. (1998). Renovación y actualidad en Educación. Reflexiones, 68, marzo. 